\title{
Stability of cubic and quartic functional equations in non-Archimedean spaces
}

\author{
M. Bavand Savadkouhi and M. Eshaghi Gordji \\ Department of Mathematics, Semnan University, \\ P. O. Box 35195-363, Semnan, Iran \\ e-mail: bavand.m@gmail.com \& madjid.eshaghi@gmail.com
}

\begin{abstract}
We prove generalized Hyres-Ulam-Rassias stability of the cubic functional equation $f(k x+y)+f(k x-y)=k[f(x+y)+f(x-y)]+2\left(k^{3}-k\right) f(x)$ for all $k \in \mathbb{N}$ and the quartic functional equation $f(k x+y)+f(k x-y)=k^{2}[f(x+y)+f(x-y)]+2 k^{2}\left(k^{2}-\right.$ 1) $f(x)-2\left(k^{2}-1\right) f(y)$ for all $k \in \mathbb{N}$ in non-Archimedean normed spaces.
\end{abstract}

\section{INTRODUCTION}

A classical question in the theory of functional equations is the following: "When is it true that a function which approximately satisfies a functional equation $\epsilon$ must be close to an exact solution of $\epsilon$ ?"

If the problem accepts a solution, we say that the equation $\epsilon$ is stable. The first stability problem concerning group homomorphisms was raised by Ulam 25] in 1940.

We are given a group $G$ and a metric group $G^{\prime}$ with metric $d(.,$.$) . Given \epsilon>0$, does there exist a $\delta>0$ such that if $f: G \rightarrow G^{\prime}$ satisfies $d(f(x y), f(x) f(y))<\delta$ for all $x, y \in G$, then a homomorphism $h: G \rightarrow G^{\prime}$ exists with $d(f(x), h(x))<\epsilon$ for all $x \in G$ ?

Ulam's problem was partially solved by Hyers 10 in 1941 . Let $E_{1}$ be a normed space, $E_{2}$ a Banach space and suppose that the mapping $f: E_{1} \rightarrow E_{2}$ satisfies the inequality

$$
\|f(x+y)-f(x)-f(y)\| \leq \epsilon \quad\left(x, y \in E_{1}\right),
$$

where $\epsilon>0$ is a constant. Then the limit $T(x)=\lim _{n \rightarrow \infty} 2^{-n} f\left(2^{n} x\right)$ exists for each $x \in E_{1}$ and $T$ is the unique additive mapping satisfying

$$
\|f(x)-T(x)\| \leq \epsilon
$$

for all $x \in E_{1}$. Also, if for each $x$ the function $t \longmapsto f(t x)$ from $\mathbb{R}$ to $E_{2}$ is continuous on $\mathbb{R}$, then $T$ is linear. If $f$ is continuous at a single point of $E_{1}$, then $T$ is continuous everywhere in $E_{1}$. Moreover (1.1) is sharp.

In 1978, Th. M. Rassias [21] formulated and proved the following theorem, which implies Hyers' theorem as a special case. Suppose that $E$ and $F$ are real normed spaces with $F$ a complete normed space, $f: E \rightarrow F$ is a mapping such that for each fixed $x \in E$ the mapping $t \longmapsto f(t x)$ is continuous on $\mathbb{R}$, and let there exist $\epsilon>0$ and $p \in[0,1)$ such that

$$
\|f(x+y)-f(x)-f(y)\| \leq \epsilon\left(\|x\|^{p}+\|y\|^{p}\right)
$$

${ }^{0} 2000$ Mathematics Subject Classification. 39B22, 39B82, 46S10.

${ }^{0}$ Keywords: Generalized Hyers-Ulam-Rassias stability; Cubic functional equation; Quartic functional equation; Non-Archimedean space; p-adic. 
for all $x, y \in E$. Then there exists a unique linear mapping $T: E \rightarrow F$ such that

$$
\|f(x)-T(x)\| \leq \frac{\epsilon\|x\|^{p}}{\left(1-2^{p-1}\right)}
$$

for all $x \in E$. The case of the existence of a unique additive mapping had been obtained by $T$. The terminology Hyers-Ulam stability originates from these historical backgrounds. The terminology can also be applied to the case of other functional equations. For more detailed definitions of such terminologies, we can refer to [5, 7], 11] and [12. In 1994, P. Găvruta [6] provided a further generalization of Th. M. Rassias' theorem in which he replaced the bound $\epsilon\left(\|x\|^{p}+\|y\|^{p}\right)$ in (1.2) by a general control function $\varphi(x, y)$ for the existence of a unique linear mapping.

The functional equation $f(x+y)+f(x-y)=2 f(x)+2 f(y)$ is called the quadratic functional equation. In particular, every solution of the quadratic functional equation is said to be a quadratic mapping, see 20, 22. A generalized Hyers-Ulam stability problem for the quadratic functional equation was proved by Skof [24] for mappings $f: X \rightarrow Y$, where $X$ is a normed space and $Y$ is a Banach space. Cholewa [3] noticed that the theorem of Skof is still true if the relevant domain $X$ is replaced by an Abelian group. In 4], Czerwik proved the generalized Hyers-Ulam stability of the quadratic functional equation. Borelli and Forti 2] generalized the stability result as follows (cf. [17, 18]): Let $G$ be an Abelian group, and $X$ a Banach space. Assume that a mapping $f: G \rightarrow X$ satisfies the functional inequality

$$
\|f(x+y)+f(x-y)-2 f(x)-2 f(y)\| \leq \varphi(x, y)
$$

for all $x, y \in G$, and $\varphi: G \times G \rightarrow[0, \infty)$ is a function such that

$$
\Phi(x, y):=\sum_{i=0}^{\infty} \frac{1}{4^{i+1}} \varphi\left(2^{i} x, 2^{i} y\right)<\infty
$$

for all $x, y \in G$. Then there exists a unique quadratic mapping $Q: G \rightarrow X$ with the property $\|f(x)-Q(x)\| \leq \Phi(x, x)$ for all $x \in G$.

Jun and Kim 13. introduced the following cubic functional equation

$$
f(2 x+y)+f(2 x-y)=2 f(x+y)+2 f(x-y)+12 f(x)
$$

and they established the general solution and the generalized Hyers-Ulam-Rassias stability for the functional equation (1.3). The function $f(x)=x^{3}$ satisfies the functional equation (1.3), which is thus called a cubic functional equation. Every solution of the cubic functional equation is said to be a cubic function. Now we introduce the following cubic functional equation

$$
f(k x+y)+f(k x-y)=k[f(x+y)+f(x-y)]+2\left(k^{3}-k\right) f(x)
$$

for all $k \in \mathbb{N}$.

In [19, Won-Gil Park and Jae-Hyeong Bae, introduced the following functional equation

$$
f(x+2 y)+f(x-2 y)=4[[f(x+y)+f(x-y)]+24 f(y)-6 f(x)
$$

and they established the general solution of the functional equation (1.5). It is easy to see that the function $f(x)=c x^{4}$ is a solution of the functional equation (1.5). Thus, it is natural that (1.5) is called a quartic functional equation and every solution of the quartic functional equation is said to be a quartic mapping. In this paper, we introduce the following quartic functional equation

$$
f(k x+y)+f(k x-y)=k^{2}[f(x+y)+f(x-y)]+2 k^{2}\left(k^{2}-1\right) f(x)-2\left(k^{2}-1\right) f(y)
$$

for all $k \in \mathbb{N}$. For more detailed definitions of such terminologies, we can refer to [16].

By a non-Archimedean field we mean a field $K$ equipped with a function (valuation) $|$.$| from$ 
$K$ into $[0, \infty)$ such that $|r|=0$ if and only if $r=0,|r s|=|r||s|$, and $|r+s| \leq \max \{|r|,|s|\}$ for all $r, s \in K$. Clearly $|1|=|-1|=1$ and $|n| \leq 1$ for all $n \in N$.

Definition 1.1. Let $X$ be a vector space over a scalar field $K$ with a non-Archimedean non-trivial valuation |.|. A function $\|\|:. X \rightarrow \mathbb{R}$ is a non-Archimedean norm (valuation) if it satisfies the following conditions:

(i) $\|x\|=0$ if and only if $x=0$;

(ii) $\|r x\|=|r|\|x\|$ for all $r \in K, x \in X$;

(iii) the strong triangle inequality (ultrametric); namely,

$$
\|x+y\| \leq \max \{\|x\|,\|y\|\} .
$$

for all $x, y \in X$. Then $(X,\|\|$.$) is called a non-Archimedean space.$

Due to the fact that

$$
\left\|x_{n}-x_{m}\right\| \leq \max \left\{\left\|x_{j+1}-x_{j}\right\|: m \leq j \leq n-1\right\} \quad(n>m)
$$

a sequence $\left\{x_{n}\right\}$ is Cauchy if and only if $\left\{x_{n+1}-x_{n}\right\}$ converges to zero in a non-Archimedean space. By a complete non-Archimedean space we mean one in which every Cauchy sequence is convergent.

In 1897, Hensel 9] discovered the p-adic numbers as a number theoretical analogue of power series in complex analysis. Fix a prime number $p$. For any nonzero rational number $x$, there exists a unique integer $n_{x} \in \mathbb{Z}$ such that $x=\frac{a}{b} p^{n_{x}}$, where $a$ and $b$ are integers not divisible by $p$. Then $|x|_{p}:=p^{-n_{x}}$ defines a non-Archimedean norm on $\mathbb{Q}$. The completion of $\mathbb{Q}$ with respect to the metric $d(x, y)=|x-y|_{p}$ is denoted by $\mathbb{Q}_{p}$ which is called the p-adic number field. In fact, $\mathbb{Q}_{p}$ is the set of all formal series $x=\sum_{k \geq n_{x}}^{\infty} a_{k} p^{k}$, where $\left|a_{k}\right| \leq p-1$ are integers. The addition and multiplication between any two elements of $\mathbb{Q}_{p}$ are defined naturally. The norm $\left|\sum_{k \geq n_{x}}^{\infty} a_{k} p^{k}\right|_{p}=p^{-n_{x}}$ is a non-Archimedean norm on $\mathbb{Q}_{p}$ and it makes $\mathbb{Q}_{p}$ a locally compact field; see [8, 23].

During the last three decades p-adic numbers have gained the interest of physicists for their research in particular in problems coming from quantum physics, p-adic strings and superstrings (cf. [14]). A key property of p-adic numbers is that they do not satisfy the Archimedean axiom: for all $x, y>0$, there exists an integer $n$ such that $x<n y$. "It is very difficult to imagine a situation where this axiom does not hold, but in fact the very space and time we inhabit have both been shown by 20th century science to be unequivocally non-Archimedean: The Archimedean axiom breaks down at the Planck scale, that is, for distances less than $1.6 \times 10^{-33}$ metres and durations less than $5.4 \times 10^{-44}$ seconds. Despite our entrenched belief that space and time are continuous, homogeneous, infinitely divisible quantities, we are now confronted with the fact that below this scale, distances and durations cannot scaled up in order to produce macroscopic distances and durations. Equivalently, we cannot meaningfully measure distances or durations below this scale. So a suggestion emerges to abandon the Archimedean axiom at very small distances. This leads to a non-Euclidean and non-Riemannian geometry of space at small distances"; cf. [26].

In [1], the authors investigated stability of approximate additive mappings $f: \mathbb{Q}_{p} \rightarrow \mathbb{R}$. They showed that if $f: \mathbb{Q}_{p} \rightarrow \mathbb{R}$ is a continuous mapping for which there exists a fixed $\epsilon$ such that $|f(x+y)-f(x)-f(y)| \leq \epsilon$ for all $x, y \in Q_{p}$, then there exists a unique additive mapping $T: \mathbb{Q}_{p} \rightarrow \mathbb{R}$ such that $|f(x)-T(x)| \leq \epsilon$ for all $x \in \mathbb{Q}_{p}$.

M. S. Moslehian and Th. M. Rassias [15] proved the generalized Hyers-Ulam stability of the Cauchy functional equation and the quadratic functional equation in non-Archimedean normed spaces.

In this paper, we solve the stability problem for cubic and quartic functional equations when 
the unknown function is one with values in a non-Archimedean space, in particular in the field of p-adic numbers.

\section{Stability of the CUbic functional EQUAtion}

In this section, we prove the generalized Hyers-Ulam-Rassias stability of the cubic functional equation (1.4). Throughout this section, we assume that $G$ is an additive group and $X$ is a complete non-Archimedean space. Now before taking up the main subject, given $f: G \rightarrow X$, we define the difference operator

$$
D f(x, y)=f(k x+y)+f(k x-y)-k[f(x+y)+f(x-y)]-2\left(k^{3}-k\right) f(x)
$$

for all $x, y \in G$ and all $k \in \mathbb{N}$. We consider the following functional inequality:

$$
\|D f(x, y)\| \leq \varphi(x, y)
$$

for an upper bound $\varphi: G \times G \rightarrow[0, \infty)$.

Theorem 2.1. Let $\varphi: G \times G \rightarrow[0, \infty)$ be a function such that

$$
\lim _{n \rightarrow \infty} \frac{\varphi\left(k^{n} x, k^{n} y\right)}{|k|^{3 n}}=0
$$

for all $x, y \in G, k \in \mathbb{N}$ and let for each $x \in G$ the limit

$$
\lim _{n \rightarrow \infty} \max \left\{\frac{\varphi\left(k^{j} x, 0\right)}{|k|^{3 j}}: 0 \leq j<n\right\},
$$

denoted by $\tilde{\varphi}(x)$, exists. Suppose that $f: G \rightarrow X$ is a mapping satisfying

$$
\|D f(x, y)\| \leq \varphi(x, y)
$$

for all $x, y \in G$. Then there exists a cubic mapping $C: G \rightarrow X$ such that

$$
\|C(x)-f(x)\| \leq \frac{1}{\left|2 k^{3}\right|} \tilde{\varphi}(x)
$$

for all $x \in G$, if

$$
\lim _{i \rightarrow \infty} \lim _{n \rightarrow \infty} \max \left\{\frac{\varphi\left(k^{j} x, 0\right)}{|k|^{3 j}}: i \leq j<n+i\right\}=0
$$

then $C$ is the unique cubic mapping satisfying (2.4).

Proof. Putting $y=0$ in (2.3), we get

$$
\left\|\frac{1}{k^{3}} f(k x)-f(x)\right\| \leq \frac{1}{\left|2 k^{3}\right|} \varphi(x, 0)
$$

for all $x \in G$ and all $k \in \mathbb{N}$. Replacing $x$ by $k^{n-1} x$ in (2.5), we get

$$
\left\|\frac{1}{k^{3 n}} f\left(k^{n} x\right)-\frac{1}{k^{3(n-1)}} f\left(k^{n-1} x\right)\right\| \leq \frac{\varphi\left(k^{n-1} x, 0\right)}{\left|2 k^{3 n}\right|}
$$

for all $x \in G$ and all $k \in \mathbb{N}$. It follows from (2.6) and (2.1) that the sequence $\left\{\frac{f\left(k^{n} x\right)}{k^{3 n}}\right\}$ is Cauchy. Since $X$ is complete, we conclude that $\left\{\frac{f\left(k^{n} x\right)}{k^{3 n}}\right\}$ is convergent. Set $C(x):=$ $\lim _{n \rightarrow \infty} \frac{f\left(k^{n} x\right)}{k^{3 n}}$. Using induction one can show that

$$
\left\|\frac{f\left(k^{n} x\right)}{k^{3 n}}-f(x)\right\| \leq \frac{1}{\left|2 k^{3}\right|} \max \left\{\frac{\varphi\left(k^{i} x, 0\right)}{|k|^{3 i}}: 0 \leq i<n\right\}
$$


for all $n \in \mathbb{N}$ and all $x \in G$. By taking $n$ to approach infinity in (2.7) and using (2.2), we obtain (2.4). By (2.1) and (2.3), we get

$$
\|D C(x, y)\|=\lim _{n \rightarrow \infty} \frac{1}{\left|k^{3 n}\right|}\left\|f\left(k^{n} x, k^{n} y\right)\right\| \leq \lim _{n \rightarrow \infty} \frac{\varphi\left(k^{n} x, k^{n} y\right)}{|k|^{3 n}}=0
$$

for all $x, y \in G$ and all $k \in \mathbb{N}$. Therefore the mapping $C: G \rightarrow X$ satisfies (1.4). If $C^{\prime}$ is another cubic mapping satisfying (2.4), then

$$
\begin{aligned}
\left\|C(x)-C^{\prime}(x)\right\| & =\lim _{i \rightarrow \infty}|k|^{-3 i}\left\|C\left(k^{i} x\right)-C^{\prime}\left(k^{i} x\right)\right\| \\
& \leq \lim _{i \rightarrow \infty}|k|^{-3 i} \max \left\{\left\|C\left(k^{i} x\right)-f\left(k^{i} x\right)\right\|,\left\|f\left(k^{i} x\right)-C^{\prime}\left(k^{i} x\right)\right\|\right\} \\
& \leq \frac{1}{\left|2 k^{3}\right|} \lim _{i \rightarrow \infty} \lim _{n \rightarrow \infty} \max \left\{\frac{\varphi\left(k^{j} x, 0\right)}{|k|^{3 j}}: i \leq j<n+i\right\} \\
& =0 .
\end{aligned}
$$

for all $x \in G$ and all $k \in \mathbb{N}$. Therefore $C=C^{\prime}$. This completes the proof of the uniqueness of $C$.

Corollary 2.2. Let $\alpha:[0, \infty) \rightarrow[0, \infty)$ be a function satisfying

(i) $\alpha(|k| t) \leq \alpha(|k|) \alpha(t)$ for all $t \geq 0$,

(ii) $\alpha(|k|)<|k|^{3}$.

Let $\delta>0$, let $G$ be a normed space and let $f: G \rightarrow X$ fulfill the inequality

$$
\|D f(x, y)\| \leq \delta[\alpha(\|x\|)+\alpha(\|y\|)]
$$

for all $x, y \in G$. Then there exists a unique cubic mapping $C: G \rightarrow X$ such that

$$
\|f(x)-C(x)\| \leq \frac{1}{\left|2 k^{3}\right|} \delta \alpha(\|x\|)
$$

for all $x \in G$ and all $k \in \mathbb{N}$.

Proof. Defining $\varphi: G \times G \rightarrow[0, \infty)$ by $\varphi(x, y):=\delta[\alpha(\|x\|)+\alpha(\|y\|)]$ we have

$$
\lim _{n \rightarrow \infty} \frac{\varphi\left(k^{n} x, k^{n} y\right)}{|k|^{3 n}} \leq \lim _{n \rightarrow \infty}\left(\frac{\alpha(|k|)}{|k|^{3}}\right)^{n} \varphi(x, y)=0
$$

for all $x, y \in G$ and all $k \in \mathbb{N}$. We have

$$
\tilde{\varphi}(x)=\lim _{n \rightarrow \infty} \max \left\{\frac{\varphi\left(k^{j} x, 0\right)}{|k|^{3 j}}: 0 \leq j<n\right\}=\varphi(x, 0)
$$

and

$$
\lim _{i \rightarrow \infty} \lim _{n \rightarrow \infty} \max \left\{\frac{\varphi\left(k^{j} x, 0\right)}{|k|^{3 j}}: i \leq j<n+i\right\}=\lim _{i \rightarrow \infty} \frac{\varphi\left(k^{i} x, 0\right)}{|k|^{3 i}}=0
$$

for all $x \in G$ and all $k \in \mathbb{N}$.

Remark 2.3. The classical example of the function $\alpha$ is the mapping $\alpha(t)=t^{p}$ for all $t \in[0, \infty)$, where $p>3$ with the further assumption that $|k|<1$.

Remark 2.4. We can formulate similar statements to Theorem 2.1 in which we can define the sequence $C(x):=\lim _{n \rightarrow \infty} k^{3 n} f\left(\frac{x}{k^{n}}\right)$ under suitable conditions on the function $\varphi$ then obtain similar result to Corollary 2.2 for $p<3$. 


\section{Stability of The QUARTIC FUnCTIONAL EQUATION}

In this section, we prove the generalized Hyers-Ulam-Rassias stability of the quartic functional equation (1.6). Throughout this section, we assume that $G$ is an additive group and $X$ is a complete non-Archimedean space. Now before taking up the main subject, given $f: G \rightarrow X$, we define the difference operator

$\Delta f(x, y)=f(k x+y)+f(k x-y)=k^{2}[f(x+y)+f(x-y)]+2 k^{2}\left(k^{2}-1\right) f(x)-2\left(k^{2}-1\right) f(y)$ for all $x, y \in G$ and all $k \in \mathbb{N}$. we consider the following functional inequality:

$$
\|\Delta f(x, y)\| \leq \psi(x, y)
$$

for an upper bound $\psi: G \times G \rightarrow[0, \infty)$.

Theorem 3.1. Let $\psi: G \times G \rightarrow[0, \infty)$ be a function such that

$$
\lim _{n \rightarrow \infty} \frac{\psi\left(k^{n} x, k^{n} y\right)}{|k|^{4 n}}=0
$$

for all $x, y \in G, k \in \mathbb{N}$ and let for each $x \in G$ the limit

$$
\lim _{n \rightarrow \infty} \max \left\{\frac{\psi\left(k^{j} x, 0\right)}{|k|^{4 j}}: 0 \leq j<n\right\},
$$

denoted by $\tilde{\psi}(x)$, exists. Suppose that $f: G \rightarrow X$ is a mapping satisfying $f(0)=0$ and

$$
\|\Delta f(x, y)\| \leq \psi(x, y)
$$

for all $x, y \in G$. Then there exists a quartic mapping $Q: G \rightarrow X$ such that

$$
\|Q(x)-f(x)\| \leq \frac{1}{\left|2 k^{4}\right|} \tilde{\psi}(x)
$$

for all $x \in G$, if

$$
\lim _{i \rightarrow \infty} \lim _{n \rightarrow \infty} \max \left\{\frac{\psi\left(k^{j} x, 0\right)}{|k|^{4 j}}: i \leq j<n+i\right\}=0
$$

then $Q$ is the unique quartic mapping satisfying (3.4).

Proof. Putting $y=0$ in (3.3), we get

$$
\left\|\frac{1}{k^{4}} f(k x)-f(x)\right\| \leq \frac{1}{\left|2 k^{4}\right|} \psi(x, 0)
$$

for all $x \in G$ and all $k \in \mathbb{N}$. Replacing $x$ by $k^{n-1} x$ in (3.5), we get

$$
\left\|\frac{1}{k^{4 n}} f\left(k^{n} x\right)-\frac{1}{k^{4(n-1)}} f\left(k^{n-1} x\right)\right\| \leq \frac{\psi\left(k^{n-1} x, 0\right)}{\left|2 k^{4 n}\right|}
$$

for all $x \in G$ and all $k \in \mathbb{N}$. It follows from (3.6) and (3.1) that the sequence $\left\{\frac{f\left(k^{n} x\right)}{k^{4 n}}\right\}$ is Cauchy. Since $X$ is complete, we conclude that $\left\{\frac{f\left(k^{n} x\right)}{k^{4 n}}\right\}$ is convergent. Set $Q(x):=$ $\lim _{n \rightarrow \infty} \frac{f\left(k^{n} x\right)}{k^{4 n}}$. From the inequality (3.5) we use iterative methods and induction on $n$ to prove our next relation:

$$
\left\|\frac{f\left(k^{n} x\right)}{k^{4 n}}-f(x)\right\| \leq \frac{1}{\left|2 k^{4}\right|} \max \left\{\frac{\psi\left(k^{i} x, 0\right)}{|k|^{4 i}}: 0 \leq i<n\right\}
$$

for all $n \in \mathbb{N}$ and all $x \in G$. By taking $n$ to approach infinity in (3.7) and using (3.2), we obtain (3.4). By (3.1) and (3.3), we get

$$
\|\Delta Q(x, y)\|=\lim _{n \rightarrow \infty} \frac{1}{\left|k^{4 n}\right|}\left\|f\left(k^{n} x, k^{n} y\right)\right\| \leq \lim _{n \rightarrow \infty} \frac{\psi\left(k^{n} x, k^{n} y\right)}{|k|^{4 n}}=0
$$


for all $x, y \in G$ and all $k \in \mathbb{N}$. Therefore the mapping $Q: G \rightarrow X$ satisfies (1.6). To prove the uniqueness property of $Q$, let $Q^{\prime}$ be another quartic mapping satisfies (3.4), then

$$
\begin{aligned}
\left\|Q(x)-Q^{\prime}(x)\right\| & =\lim _{i \rightarrow \infty}|k|^{-4 i}\left\|Q\left(k^{i} x\right)-Q^{\prime}\left(k^{i} x\right)\right\| \\
& \leq \lim _{i \rightarrow \infty}|k|^{-4 i} \max \left\{\left\|Q\left(k^{i} x\right)-f\left(k^{i} x\right)\right\|,\left\|f\left(k^{i} x\right)-Q^{\prime}\left(k^{i} x\right)\right\|\right\} \\
& \leq \frac{1}{\left|2 k^{4}\right|} \lim _{i \rightarrow \infty} \lim _{n \rightarrow \infty} \max \left\{\frac{\psi\left(k^{j} x, 0\right)}{|k|^{4 j}}: i \leq j<n+i\right\} \\
& =0 .
\end{aligned}
$$

for all $x \in G$ and all $k \in \mathbb{N}$. Therefore $Q=Q^{\prime}$. This completes the proof of the uniqueness of $Q$.

Corollary 3.2. Let $\beta:[0, \infty) \rightarrow[0, \infty)$ be a function satisfying

(i) $\beta(|k| t) \leq \beta(|k|) \beta(t)$ for all $t \geq 0$,

(ii) $\beta(|k|)<|k|^{4}$.

Let $\delta>0$, let $G$ be a normed space and let $f: G \rightarrow X$ fulfill the inequality

$$
\|\Delta f(x, y)\| \leq \delta[\beta(\|x\|)+\beta(\|y\|)]
$$

for all $x, y \in G$. Then there exists a unique quartic mapping $Q: G \rightarrow X$ such that

$$
\|f(x)-Q(x)\| \leq \frac{1}{\left|2 k^{4}\right|} \delta \beta(\|x\|)
$$

for all $x \in G$ and all $k \in \mathbb{N}$.

Proof. Putting $\psi(x, y):=\delta[\beta(\|x\|)+\beta(\|y\|)]$ in above theorem. We have

$$
\lim _{n \rightarrow \infty} \frac{\psi\left(k^{n} x, k^{n} y\right)}{|k|^{4 n}} \leq \lim _{n \rightarrow \infty}\left(\frac{\beta(|k|)}{|k|^{4}}\right)^{n} \psi(x, y)=0
$$

for all $x, y \in G$ and all $k \in \mathbb{N}$. We have

$$
\tilde{\psi}(x)=\lim _{n \rightarrow \infty} \max \left\{\frac{\psi\left(k^{j} x, 0\right)}{|k|^{4 j}}: 0 \leq j<n\right\}=\psi(x, 0)
$$

and

$$
\lim _{i \rightarrow \infty} \lim _{n \rightarrow \infty} \max \left\{\frac{\psi\left(k^{j} x, 0\right)}{|k|^{4 j}}: i \leq j<n+i\right\}=\lim _{i \rightarrow \infty} \frac{\psi\left(k^{i} x, 0\right)}{|k|^{4 i}}=0
$$

for all $x \in G$ and all $k \in \mathbb{N}$.

Remark 3.3. The classical example of the function $\beta$ is the mapping $\beta(t)=t^{p}$ for all $t \in[0, \infty)$, where $p>4$ with the further assumption that $|k|<1$.

Remark 3.4. We can formulate similar statements to Theorem 3.1 in which we can define the sequence $Q(x):=\lim _{n \rightarrow \infty} k^{4 n} f\left(\frac{x}{k^{n}}\right)$ under suitable conditions on the function $\psi$ then obtain similar result to Corollary 3.2 for $p<4$.

\section{REFERENCES}

[1] L. M. Arriola and W. A. Beyer, Stability of the Cauchy functional equation over p-adic fields, Real Analysis Exchange. 31 (2005/2006), 125-132.

[2] C. Borelli and G. L. Forti, On a general Hyers-Ulam stability result, Internat. J. Math. Math. Sci. 18 (1995), 229-236.

[3] P. W. Cholewa, Remarks on the stability of functional equations, Aequationes Math. 27 (1984), 76-86. 
[4] S. Czerwik, On the stability of the quadratic mapping in normed spaces. Abh. Math. Sem. Univ. Hamburg. 62 (1992), 59-64.

[5] Z. Gajda, On stability of additive mappings. Internat. J. Math. Math. Sci. 14 (1991), 431-434.

[6] P. Găvruta, A generalization of the Hyers-Ulam-Rassias stability of approximately additive mappings, J. Math. Anal. Appl. 184 (1994), 431-436.

[7] A. Grabiec, The generalized Hyers-Ulam stability of a class of functional equations, Publ. Math. Debrecen 48 (1996), 217-235.

[8] F. Q. Gouveaa, p-adic Numbers. Springer-Verlag, Berlin. 1997.

[9] K. Hensel, Über eine neue Begründung der Theorie der algebraischen Zahlen. Jahresber. Deutsch. Math. Verein. 6 (1897), 83-88.

[10] D. H. Hyers, On the stability of the linear functional equation, Proc. Nat. Acad. Sci. USA 27 (1941), 222-224.

[11] D. H. Hyers, G. Isac and Th. M. Rassias, Stability of Functional Equations in Several Variables, Birkhäuer, Basel. 1998.

[12] S. M. Jung, Hyers-Ulam-Rassias Stability of Functional Equations in Mathematical Analysis. Hadronic Press lnc. Palm Harbor, Florida. 2001.

[13] K. W. Jung and H. M. Kim, The generalized Hyers-Ulam-Rassias stability of a cubic functional equation, J. Math. Anal. Appl. 274 (2002), no. 2, 267-278.

[14] A. Khrennikov, Non-Archimedean Analysis, Quantum Paradoxes, Dynamical Systems and Biological Models. Kluwer Academic Publishers, Dordrecht. 1997.

[15] M. S. Moslehian and Th. M. Rassias, Stability of functional equations in nonArchimedean spaces, Applicable Analysis and Discrete Mathematics. 1 (2007), 325-334.

[16] A. Najati, On the stability of a quartic functional equation, J. Math. Anal. Appl. 340 (2008), no. 1, 569-574.

[17] C. Park, Generalized quadratic mappings in several variables. Nonlinear Anal. TMA. 57 (2004), 713-722.

[18] C. Park, On the stability of the quadratic mapping in Banach modules. J. Math. Anal. Appl. 276 (2002), 135-144.

[19] W. G. Park and J. H. Bae, On the stability a bi-quartic functional equation, Nonlinear Anal. 62 (2005), no. 4, 643-654.

[20] Th. M. Rassias (Ed.), Functional Equations and Inequalities,Kluwer Academic, Dordrecht, 2000.

[21] Th. M. Rassias, On the stability of the linear mapping in Banach spaces, Proc. Amer. Math. Soc. 72 (1978), 297-300.

[22] Th. M. Rassias, On the stability of the quadratic functional equation and its applications. Studia Univ. Babes-Bolyai. 43 (1998), 89-124.

[23] A. M. Robert, A Course in p-adic Analysis. SpringerVerlag, New York. 2000.

[24] F. Skof, Proprietá localie approssimazione dioperatori, Rend. Sem. Mat. Fis. Milano. 53 (1983), 113-129.

[25] S. M. Ulam, A Collection of Mathematical Problems, Interscience Tracts in Pure and Applied Mathematics, Interscience Publisher, New York, 1960.

[26] V. S. Vladimirov, I. V. Volovich and E. I. Zelenov, p-adic Analysis and Mathematical Physics. World Scientific. 1994. 